\title{
Effectiveness of a Multimodal Digital Psychotherapy Platform for Adult Depression: A Naturalistic Feasibility Study
}

Enitan T Marcelle ${ }^{1}$, BA; Laura Nolting ${ }^{2}$, BS; Stephen P Hinshaw ${ }^{1,3}, \mathrm{PhD}$; Adrian Aguilera ${ }^{4,5}, \mathrm{PhD}$

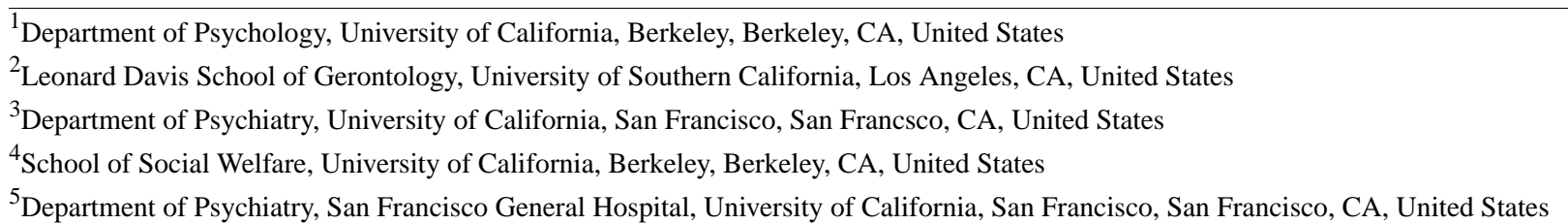

\section{Corresponding Author:}

Enitan T Marcelle, BA

Department of Psychology

University of California, Berkeley

Tolman Hall

Berkeley, CA,

United States

Phone: 12164968894

Email: emarcelle@berkeley.edu

\begin{abstract}
Background: Although psychotherapy is one of the most efficacious and effective treatments for depression, limited accessibility to trained providers markedly limits access to care. In an attempt to overcome this obstacle, several platforms seeking to provide these services using digital modalities (eg, video, text, and chat) have been developed. However, the use of these modalities individually poses barriers to intervention access and acceptability. Multimodal platforms, comprising those that allow users to select from a number of available modalities, may be able to provide a solution to these concerns.
\end{abstract}

Objective: We aimed to investigate the preliminary effectiveness of providing psychotherapy through a multimodal digital psychotherapy platform. In addition, we aimed to examine differential responses to intervention by gender, self-reported physical health status, and self-reported financial status, as well as how prior exposure to traditional face-to-face psychotherapy affected the effectiveness of a multimodal digital psychotherapy intervention. Finally, we aimed to examine the dose-response effect.

Methods: Data were collected from a total of 318 active users of BetterHelp, a multimodal digital psychotherapy platform. Data on physical health status, financial status, and prior exposure to psychotherapy were obtained using self-report measures. Effectiveness was determined by the extent of symptom severity change, which was measured using the Patient Health Questionnaire at Time 1 (time of enrollment) and Time 2 ( 3 months after enrollment). Intervention dosage was measured as the sum of individual therapist-user interactions across modalities.

Results: Depression symptom severity was significantly reduced after the use of the multimodal digital psychotherapy intervention $(P<.001)$. Individuals without prior traditional psychotherapy experience revealed increased improvement after intervention $(P=.006)$. We found no significant dose-response effect of therapy, nor significant differences in outcomes across gender, self-reported financial status, and self-reported physical health status.

Conclusions: Users of BetterHelp experienced significantly reduced depression symptom severity after engaging with the platform. Study findings suggest that this intervention is equally effective across gender, self-reported financial status, and self-reported physical health status and particularly effective for individuals without a history of psychotherapy. Overall, study results suggest that multimodal digital psychotherapy is a potentially effective treatment for adult depression; nevertheless, experimental trials are needed. We discuss directions for future research.

(JMIR Mhealth Uhealth 2019;7(1):e10948) doi: 10.2196/10948

\section{KEYWORDS}

cognitive therapy; depression; digital health; live chat; mHealth; mental health; text messaging; video; mobile phone 


\section{Introduction}

Major depressive disorder is a commonly occurring condition $[1,2]$ associated with a multitude of adverse health outcomes $[3,4]$ and is projected to be the second leading cause of disability worldwide by the year 2020 [5,6]. Development and dissemination of efficacious and accessible treatment for the disorder are an area of increasing importance. Psychotherapy has been shown to be one of the most efficacious psychosocial treatments for depression $[7,8]$ and works by teaching patients cognitive strategies that enable them to target and manage undesirable thoughts, habits, and emotions underlying presenting depressive symptoms [9]. Although the literature has demonstrated the need for psychotherapy in the successful treatment of depression [10-13], limited geographical access to trained professionals remains one of the most significant barriers to traditional face-to-face care [14]. Telemental health, or the use of digital technology to provide long-distance clinical mental health care, is a field rapidly growing in response to issues of access [15-18].

Multimodal digital therapy platforms, that is, platforms that offer multiple modes of digital communication, hold promise in overcoming persisting barriers to intervention access and acceptability. In the United States, 1 in every 4 adults is in need of counseling, although only $13.4 \%$ of adults report receiving services [19]. This treatment gap is seen around the world [20] and is driven by a combination of stigma surrounding mental health support-seeking behavior [21] and limited geographical access to providers [14]. Digital psychotherapeutic interventions have been shown to increase intervention reach by providing efficacious alternatives to traditional therapy. These digital interventions increase both ease of access and anonymity of service seeking. To date, digital therapy has largely centered around the internet- (eg, videoconference or chat) and mobile (eg, short message service [SMS] text messaging or phone)-based modalities.

Extant literature demonstrates marked demographic differences in modality access and acceptance. Internet-based interventions have been shown to be efficacious [22], with a 2009 meta-analysis of 12 studies finding a mean effect size of $d=$ 0.41 for internet-based psychological treatments for adult depression [23]. This effect size was increased $(d=0.61)$ after excluding standalone interventions and considering only those that included support or guidance from a therapist. While evidence is mixed [24], research suggests that increased guidance improves the efficacy of internet-based psychotherapy [25]. Although such interventions have been found to be efficacious, barriers to accessible, reliable, and consistent internet connection keep these interventions beyond the reach of traditionally underserved populations [18]. In response to this persisting digital divide, researchers have investigated internet-free, mobile phone- and SMS text message-based therapies as a way of continuing to increase access to care [26]. Mobile phone access is considerably more ubiquitous than internet access [15,27], and the development of mobile platforms has enabled mental health professionals to provide care to an even greater percentage of individuals in need. In addition, mobile phone-based psychotherapy for adult depression has been demonstrated to be efficacious [28], yet this workaround does not come without its own set of limitations. Specifically, younger adults feel more comfortable communicating and building relationships remotely [29], although older adults prefer communication modalities that can mirror more traditional, in-person interactions, such as video calls. A multitude of studies have examined and demonstrated the efficacy and effectiveness of single- or even dual-modality digital psychotherapy platforms [22,30-34], but we know of no existing work investigating the effectiveness of a multimodal platform. We propose a multimodal psychotherapy platform that allows users to choose from internet-based video or live chat and internet-free, mobile SMS text message or phone therapy interchangeably as a potential solution to this demographic variability in intervention usability and effectiveness.

In addition to the modality, other important predictors of digital psychotherapy outcomes include demographic characteristics as well as level of engagement. The existing literature in face-to-face psychotherapy has focused on differences in outcomes between men and women, as well as on differences across ages and socioeconomic status. Despite growing product innovation, the characteristics and demographics of populations for whom digital psychotherapies do and do not work remains unknown. Although evidence of gender effects on psychotherapy outcomes is mixed, research suggests that women report an increased symptom reduction after traditional psychotherapy [35-39]. Yet research on Web-based psychotherapy suggests that men report an increased symptom reduction compared with women. Given higher rates of the stigma surrounding therapy-seeking behaviors in men [40], it has been suggested that the anonymity afforded by Web-based platforms may be an explanation for this latter finding. Research examining the effect of age on psychotherapy outcomes also remains largely inconclusive. Some evidence suggests that younger clients may respond more quickly and report greater posttreatment improvement in psychotherapy, although older adults are more likely to adhere to treatment $[7,41,42]$. The literature focusing on broader socioeconomic predictors of traditional psychotherapy acceptance and response has focused on economic status, physical health status, and experience with therapy as key predictive variables, finding positive correlations between outcomes and these factors. We speculate that prior counseling experience and higher financial or health status will predict improved digital psychotherapy outcomes as well. Although a number of researchers have examined the effect of engagement, or dose-response effect of psychotherapy, in traditional settings [43], considerably fewer studies have examined this relationship in the context of digital psychotherapy [44-46]. We additionally aimed to examine the dose-response effect of our digital psychotherapy platform.

The aim of this feasibility study was to investigate the initial effectiveness of delivering psychotherapy via BetterHelp, a multimodal internet- and mobile-based psychotherapy service provider. Bowen et al [47] defined a feasibility study as any study aiming to "determine whether an intervention is appropriate for further testing." In line with this definition, this naturalistic and quasi-experimental investigation aimed to examine individuals' responses to BetterHelp to generate useful 
data to guide and justify future randomized controlled trials. Effectiveness studies are defined as those that investigate the extent to which an intervention does more good than harm when administered in a "real-word" setting, as opposed to in ideal and highly controlled conditions $[48,49]$. In line with our aim of investigating the effectiveness of a multimodal method of delivering psychotherapy for adult patients with depression, this work defined the intervention effectiveness as a significant reduction in the depressive symptom severity among individuals using BetterHelp. We hypothesized that engagement with BetterHelp will significantly reduce depression symptom severity. In addition, in this exploratory study, we aimed to investigate the ways in which multimodal digital psychotherapy outcomes vary by subpopulation (gender, age, financial and physical status, and prior therapy experience) as well as by the level of engagement.

\section{Methods}

\section{Participants}

In total, 318 BetterHelp clients (of whom 254, 79.9\%, were females), recruited from a larger pool of active BetterHelp users, participated in this study. BetterHelp users are individuals aged $\geq 18$ years seeking to improve their quality of life. Users aged $<18$ years or under the care of a legal guardian were excluded from BetterHelp participation. Furthermore, individuals with thoughts of hurting themselves or others, those in urgent crisis or emergency situations, those diagnosed with a severe mental illness or advised to be in psychological supervision or psychiatric care, and those required to undergo therapy or counseling either by a court order or by any other authority were also excluded. BetterHelp users who met the eligibility criteria to participate in this study were invited to participate. Inclusion criteria for this study included a self-endorsed primary concern of feelings of overwhelming sadness, grief, or depression. Study participants were excluded from participation if preintervention levels of depression fell below mild clinical significance, that is, a score of $<5$ on the Patient Health Questionnaire (PHQ-9) [50], or if they had not engaged with BetterHelp for a minimum of 90 days. Users with preintervention depression in the mild to severe range, (ie, PHQ-9 scores $\geq 5$ and $\leq 27$ ) were included (Textbox 1). A total of 1148 BetterHelp users met all the eligibility criteria and were invited to participate. Our observed response rate of $27.70 \%$ (318/1148) is comparable to response rates seen in other Web-based survey studies [51]. The racial and ethnic makeup of this sample, as well as comorbid mental or physical health concerns, is unfortunately unknown as the BetterHelp platform does not currently collect this information. Ages of participants meeting the eligibility criteria ranged from 19 to 72 (mean 33.27 [SD 11.29]) years. At baseline, of 318, $119(37.4 \%)$ participants met the criteria for mild depression, $91(28.6 \%)$ for moderate depression, $75(23.6 \%)$ for moderately severe depression, and $33(10.4 \%)$ for severe depression [50,52,53] (Table 1).

Textbox 1. Inclusion and exclusion criteria for study participation.

Inclusion criteria

- $\quad$ Age $\geq 18$ years

- BetterHelp user for at least 90 days

- $\quad$ Baseline Patient Health Questionnaire score $\geq 5$ and $\leq 27$

- Self-endorsed primary concerns of feelings of overwhelming sadness, grief, or depression

Exclusion criteria

- $\quad$ Age $<18$ years

- Under the care of a legal guardian

- Thoughts of hurting self or others

- Severe mental illness

- Advised to be in psychological supervision or psychiatric care, or required to undergo therapy or counseling either by a court order or by any other authority

- BetterHelp user for $<90$ days

- $\quad$ Baseline Patient Health Questionnaire score $<5$ 
Table 1. Preintervention and postintervention Patient Health Questionnaire (PHQ-9) scores by the diagnostic category (N=318).

\begin{tabular}{llll}
\hline PHQ-9 diagnostic category & PHQ-9 score & Preintervention, $\mathrm{n}(\%)$ & Postintervention, $\mathrm{n}(\%)$ \\
\hline Minimal depression & $0-4$ & $0(0)$ & $63(19.8)$ \\
Mild depression & $5-9$ & $119(37.4)$ & $141(41.2)$ \\
Moderate depression & $10-14$ & $91(28.6)$ & $66(20.8)$ \\
Moderately severe depression & $15-19$ & $75(23.6)$ & $40(12.6)$ \\
Severe depression & $20-27$ & $33(10.4)$ & $18(5.7)$ \\
\hline
\end{tabular}

\section{Measures}

\section{Patient Health Questionnaire}

The PHQ-9 [50,52,53] is a 10-item, self-report measure inquiring about the presence of depressive symptoms in the previous 2 weeks. It is used in clinical practice to monitor depression symptoms and severity [54]. The measure probes how often a respondent has been bothered by specific problems, takes only a few minutes to complete, and is scored on a 4-point Likert scale ranging from 0 (Not At All) to 3 (Nearly Every Day). The scale has demonstrated high internal consistency (Cronbach alpha=.86 to .89) as well as excellent test-retest reliability $(r=0.84)$ [52]. Participants completed this measure at baseline and follow-up.

\section{Working Alliance Inventory-Short Revised}

The Working Alliance Inventory-Short Revised (WAI-SR) [55] is a 12-item measure assessing the quality of the therapeutic relationship. This measure of therapeutic alliance assesses the following domains: (1) agreement on the tasks of therapy; (2) agreement on the goals of therapy; and (3) development of an affective bond. The measure has consistently demonstrated good reliability (alpha>.80) as well as good convergent validity $(r>0.64)$; it was administered at follow-up to assess user rapport with therapist.

\section{Prior Exposure to Therapy}

Before beginning therapy, a binary measure was administered to assess prior exposure to psychotherapy. BetterHelp users were asked the question "Have you ever been in counseling or therapy before?" and probed to reply with either "Yes" or "No."

\section{Self-Reported Physical Health Status and Financial Status}

At baseline, BetterHelp users were asked to rate their current physical health and current financial status. Responses were scored on a 3-point Likert scale ranging from Good to Poor.

\section{Procedure}

\section{Intervention Description}

The BetterHelp psychotherapy platform is currently the largest multimodal digital psychotherapy platform available worldwide [56]. BetterHelp utilizes a preference-based approach in which users can use any and all combinations of text, video, chat, or phone communication over the course of psychotherapy, as they choose. BetterHelp procedures are as follows: before beginning therapy, clients are asked to complete questionnaires probing symptom levels, personal history, and motivation for seeking therapy. Although BetterHelp counselors vary in approach (ie, cognitive behavioral therapy, acceptance and commitment therapy, etc), each BetterHelp counselor is a required to have attained a PhD, PsyD, Marriage Family Therapist, Licensed Clinical Social Worker, Licensed Professional Counselor, or Licensed Master Social Worker-level license to practice. BetterHelp's algorithm then matches clients with an available BetterHelp counselor who best fits their objectives, counselor preferences, and needs. Preferred modality of communication is not taken into account in BetterHelp's algorithm, as BetterHelp users can utilize any form of communication at any time, and all BetterHelp counselors are required to make themselves available to provide therapy through the client's chosen modality. After the match is made, BetterHelp provides client and counselor with a dedicated "room" in which all communication takes place. Video, live chat, and phone sessions require advanced scheduling, while SMS text message exchanges do not. Multimedia Appendices 1-4 display BetterHelp platform as well as its video and live chat invitation and interface.

\section{Data Collection}

After engaging with the BetterHelp platform for 3 months, users received a notification inviting them to participate in an ongoing research study. This 3-month time window was selected to mirror the existing dose-response research in psychotherapy, suggesting that $>50 \%$ of patients are able to respond after 12.7 sessions of weekly psychotherapy [57]. BetterHelp users were allowed 2 weeks to respond to prompts inviting them to participate in research. Users who did not respond within the allotted 2 weeks were excluded from study participation. Respondents were asked to repeat the PHQ-9 as well as complete the WAI-SR. All respondents to the research invitation completed all questionnaires. BetterHelp data analysts sent relevant data to authors in a deidentified data file.

\section{Data Analysis}

All analyses were conducted in SPSS Version 25 (IBM Corp Released 2017. IBM SPSS Statistics for Macintosh, Version 25.0) and R version 2.13.1 (R Development Core Team (2011). To examine the effectiveness of delivering psychotherapy through a multimodal digital platform, we examined the percentage of users exhibiting established markers of clinical improvement, as determined by change in PHQ-9 scores [53]. We first examined the percentage of users demonstrating clinically significant improvement. We additionally examined the percentage of users demonstrating a partial response to intervention and the percentage of users qualifying as being in remission after intervention use. We then examined changes in the symptom severity among BetterHelp users from pre- to posttreatment using paired samples $t$ test. Because of our study 
design (ie, comparing mean PHQ-9 scores of BetterHelp users across 2 time-points), we determined paired samples $t$ test to be the most appropriate method of analysis [58]. Our study design and the nature of our dependent and independent variables led us to determine a one-way analysis of covariance (ANCOVA), covarying baseline symptom severity, as the most appropriate means testing the differential effects of age and gender on therapy outcomes. To examine the effect of financial status, health status, and prior exposure to therapy on treatment outcomes, we conducted a second one-way ANCOVA, again adjusting for baseline symptom severity. The effect of dosage on treatment outcomes was examined using probit dose-response regression, a method used across the extant dose-response literature to predict the amount or dose of treatment needed to achieve a desired response or effect [43].

\section{Ethics Statement}

This study was conducted in accordance with the Institutional Review Board and Office for the Protection of Human Subjects at the University of California, Berkeley. All subjects were informed of the risks and benefits of participating in the study and gave electronic informed consent to participate via the BetterHelp platform. Participants were provided with access to the BetterHelp platform and were not additionally compensated or incentivized to participate in any way. The research study was approved by the Institutional Review Board at the University of California, Berkeley.

\section{Results}

\section{Descriptive Statistics}

Table 2 describes the sociodemographic characteristics (age, gender, health or financial status, and prior exposure to therapy) of the study sample. Overall, of 318 participants, 72 (22.6\%) rated their financial status as poor, $167(52.5 \%)$ as fair, and 49 $(15.4 \%)$ as good. In addition, $24(7.5 \%)$ participants rated their health status as poor, $165(51.9 \%)$ as fair, and $118(37.1 \%)$ as good; furthermore, 91 (28.6\%) participants did not have prior exposure to counseling or therapy. Table 3 displays the modality usage in the BetterHelp user sample.

Table 2. Sociodemographic characteristics of BetterHelp users.

\begin{tabular}{|c|c|}
\hline Characteristic & BetterHelp users, n (\%) \\
\hline \multicolumn{2}{|l|}{ Gender } \\
\hline Female & 254 (79.9) \\
\hline Male & $64(20.1)$ \\
\hline \multicolumn{2}{|l|}{ Age (years) } \\
\hline $18-34$ & $200(62.6)$ \\
\hline $35-49$ & $89(27.7)$ \\
\hline$>50$ & $29(9.1)$ \\
\hline \multicolumn{2}{|c|}{ Physical health status } \\
\hline Poor & $24(7.5)$ \\
\hline Fair & $165(51.9)$ \\
\hline Good & $118(37.1)$ \\
\hline \multicolumn{2}{|c|}{ Financial status } \\
\hline Poor & $72(22.6)$ \\
\hline Fair & $167(52.5)$ \\
\hline Good & $49(15.4)$ \\
\hline \multicolumn{2}{|c|}{ Prior counseling experience } \\
\hline No & $91(28.6)$ \\
\hline Yes & $216(67.9)$ \\
\hline
\end{tabular}


Table 3. Modality usage of BetterHelp users.

\begin{tabular}{ll}
\hline Modality usage & BetterHelp user, $\mathrm{n}(\%)$ \\
\hline Short message service text message user & $318(100)$ \\
Live chat user & $53(16.7)$ \\
Phone user & $37(11.6)$ \\
Video user & $1(0.3)$ \\
Single modality user & $236(74.2)$ \\
Dual modality user & $74(23.3)$ \\
Tri modality user & $7(2.2)$ \\
All modality user & $1(0.3)$ \\
\hline
\end{tabular}

\section{Overall Effectiveness of BetterHelp}

In this study, of 318 participants, $120(37.8 \%)$ demonstrated a clinically significant improvement and 194 (62\%) demonstrated a partial response (as defined by at least a 5-point score reduction on the PHQ-9 and a postintervention score $\leq 9$, respectively [53]) after engaging with BetterHelp for 3 months; in addition, $63(19.8 \%)$ participants qualified as being in remission (as defined by a postintervention score $<5$ ) by Time 2. Paired samples $t$ test results revealed a significant decrease in symptom severity posttreatment with effect size in the medium range (pretreatment: mean 12.57 [SD 5.35]; posttreatment: mean 9.36 [SD 5.51]; $t_{317}=10.80 ; P<.001$, one-tailed; Cohen $d=0.61$; Figure 1). Mean PHQ-9 pretreatment scores reflected moderate levels of current depression, whereas mean posttreatment scores reflected mild levels of current depression, as determined by PHQ-9 clinical cutoffs [52]. As shown in Table 1, after using BetterHelp for 3 months, of 318 participants, $63(19.8 \%)$ met the criteria for minimal depression, $141(41.2 \%)$ for mild depression, $66(20.8 \%)$ for moderate depression, $40(12.6 \%)$ for moderately severe depression, and $18(5.7 \%)$ for severe depression [52,53].

\section{Demographic Influence on Outcomes}

A one-way ANCOVA examining the differential effects of age and gender on therapy outcomes, covarying baseline symptom severity, revealed no significant differences. Outcomes did not significantly differ across age $\left(F_{45,242}=0.98 ; P=.51\right)$ and gender $\left(F_{1,242}=.092 ; P=.76\right)$.

\section{Socioeconomic and Environmental Influence on Outcomes}

A one-way ANCOVA examining effects of financial status, health status, and prior exposure to therapy on treatment outcomes, covarying baseline symptom severity, revealed a significant effect of prior exposure to therapy on treatment outcome $\left(F_{1,260}=7.531 ; P=.006\right)$. Individuals with prior therapy exposure experienced significantly fewer gains after treatment compared with individuals without prior exposure (Figure 2). Treatment outcomes did not significantly differ across participant financial status $\left(F_{2,260}=1.563 ; P=.21\right)$ or health status $\left(F_{2,260}=1.575 ; P=.21\right)$.

\section{Dose-Response Effect}

Treatment dosage was measured as the sum of individual therapist-user interactions across modalities (text message, phone, video, and live chat). Response was measured as a binary variable indicating the presence or absence of clinical improvement, as defined by a PHQ- 9 score change of $\geq 5$ points. Mean number of interactions in this study was 125.3 (SD 392.5). Following the methodology laid out by Howard et al in the probit model [59], the dose corresponded to the log of the number of total interactions, to reduce skew. Because participants who did not interact with BetterHelp were not included in the sample, taking the log of 0 was not a possibility. Results revealed no significant dose-response effect $(P=.14)$, which was maintained after adding baseline severity into the model.

\section{Post-Hoc Analyses}

To adjust PHQ-9 scores for regression to the mean, an ANCOVA in which the difference between baseline and posttreatment scores was regressed onto the mean-centered baseline score was used $[58,60]$. These additional analyses also revealed a significant mean difference $(P<.001)$.

To assess the hypothesis that differences in treatment gains between individuals with and without prior therapy experience may be driven by differences in therapeutic alliance associated with prior therapy exposure, an independent samples $t$ test was performed. Cronbach alpha for the 12 WAI-SR items was high (alpha=.946). The results revealed no significant effect of prior therapy experience on WAI-SR total scores $(P=.55)$. 
Figure 1. Overall Patient Health Questionnaire (PHQ-9) pre-post change. Error bars represent SEs.

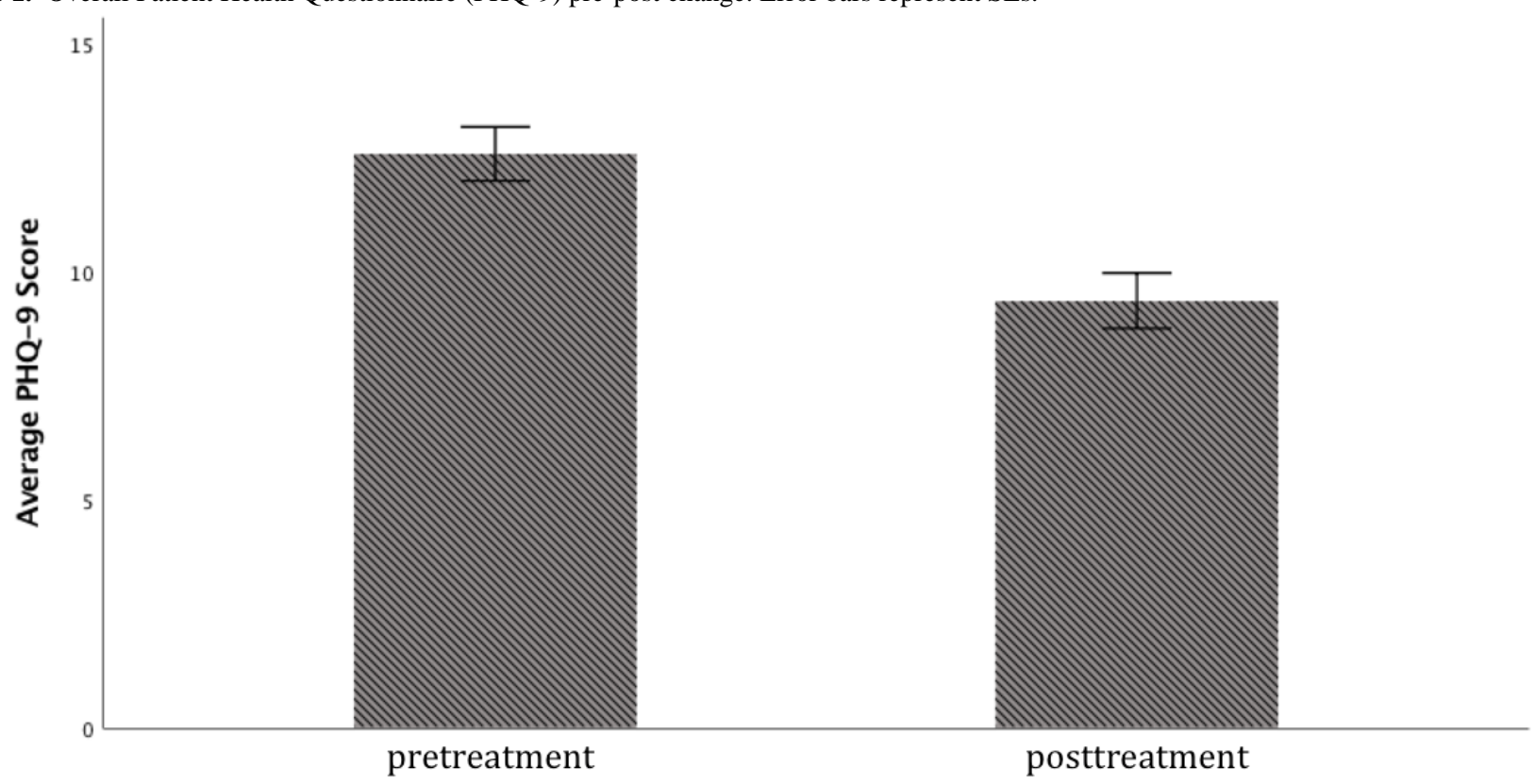

Figure 2. Difference in Patient Health Questionnaire (PHQ-9) score change by prior counseling experience status. Error bars represent SEs.

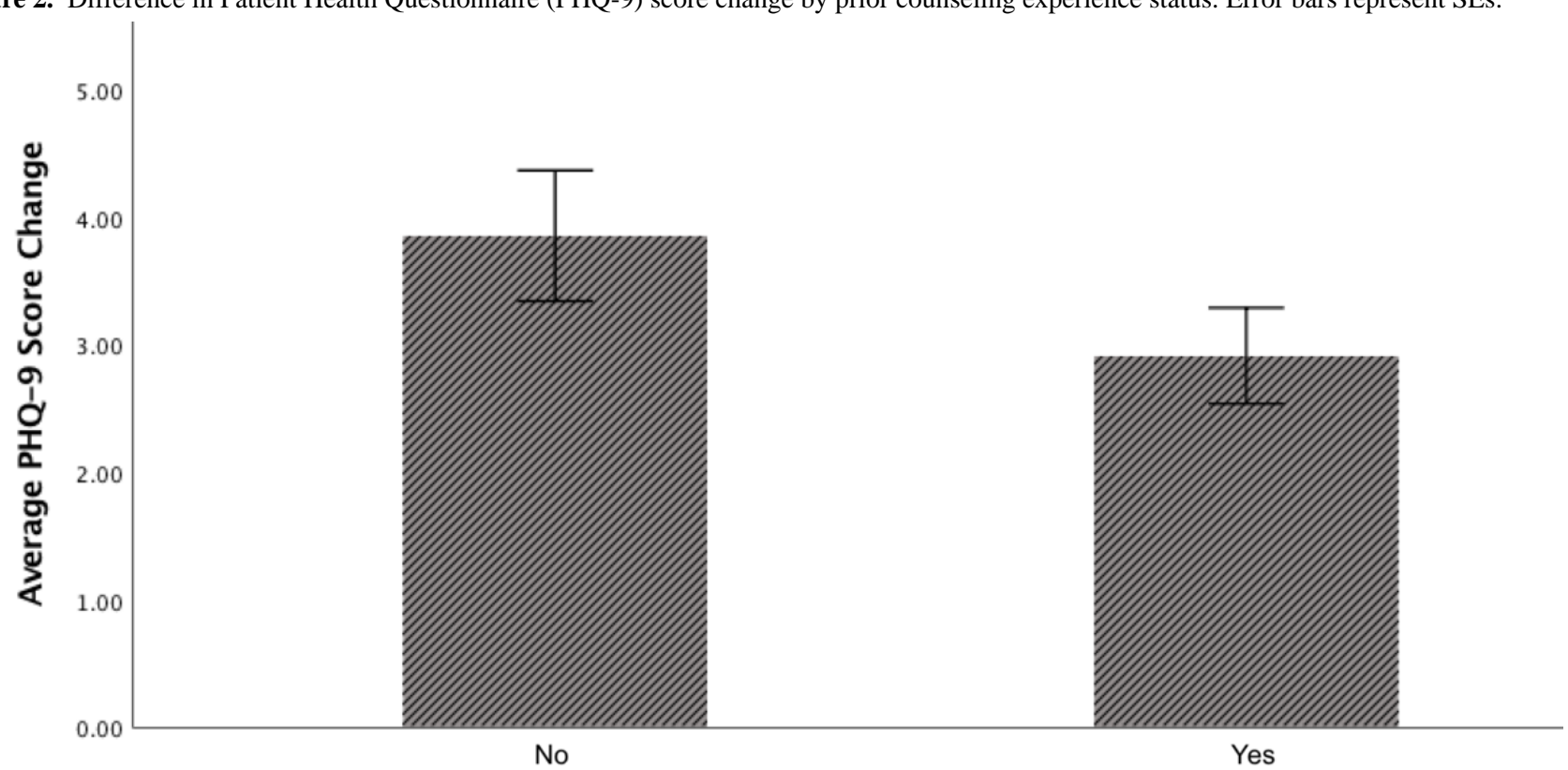

Prior Counseling Experience

\section{Discussion}

\section{Principal Findings}

Our results indicate that multimodal digital psychotherapy may be an effective treatment for adult depression. Given our preliminary results demonstrating the effectiveness of this method of psychotherapy dissemination, we believe that multimodal digital psychotherapy may hold promise in overcoming some existing issues of psychotherapy access. Hence, continued research is needed. Users of a multimodal digital psychotherapy platform experienced significantly improved self-reported symptoms after engaging with BetterHelp, with $37.8 \%$ (120/318) users experiencing clinically significant improvement in depressive symptoms within 3 months. Moreover, no significant associations were found between changes in depressive symptoms and sociodemographic variables, including age, gender, and self-reported financial or physical health status. We suspect that increased accessibility and flexibility provided by a preference-based multimodal platform may be driving these latter findings [61,62]. Although this study cannot establish causality, our findings suggest that multimodal digital psychotherapy may be an effective solution to reduce existing barriers to accessible and preference-based digital psychotherapy.

Participants who had previously engaged in traditional face-to-face psychotherapy showed significantly less symptomatic improvement compared with those who had not. Among several potential explanations, we postulate that 
individuals with prior therapy experience may present with more chronic, complex, comorbid, or treatment-resistant forms of depression requiring a higher level of care [63-65]. Post-hoc analyses did not reveal any significant association between therapeutic relationship quality (as measured by the WAI) and prior therapy engagement, suggesting that this finding is not merely related to differences in therapist rapport and alliance linked to prior treatment exposure. Future research should seek to investigate differences in outcomes across diagnostic categories, taking into account commonly comorbid conditions known to influence response to treatment such as generalized anxiety disorder, attention deficit/hyperactivity disorder, and panic disorder. Baseline evaluation of the presence and severity of additional diagnoses may ensure that individuals with comorbid conditions are matched with an appropriately specialized therapist.

No general dose-response effect was detected in this study. Although this is not the first study to find nonsignificant dose-response effects of psychotherapy, we speculate that our existing measure of dosage may disguise existing dose-response effects, given that the quantity and significance of content shared in a single message may vary greatly by individual. Future research may seek to examine effects of word count as opposed to the message count, though due to user privacy concerns, researchers were not able to access these data at this time. Furthermore, the number of days spent interacting with the BetterHelp platform may prove to be a more valid measure of engagement than total interactions.

All study participants in this preference-based study utilized the SMS text message modality. A majority of participants utilized only one therapy modality over the course of the intervention, with only a single participant making use of all 4 modalities. We hypothesize that this pattern of use is driven by the increased convenience and flexibility provided by SMS text message-based therapy (ie, a previously scheduled appointment is not needed to utilize the SMS text message-based modality, whereas an appointment is needed to utilize live chat-, phone-, or video-based therapy). Future work will seek to test this hypothesis by obtaining qualitative data from participants regarding motivations behind modality choices, as well as to elucidate the added benefit of a multimodality psychotherapy platform (ie, using a randomized "text-only" vs "multimodal" design).

\section{Limitations}

This exploratory investigation contains several limitations of note. First and foremost, we lacked a randomly assigned control group. Future investigations with appropriate controls, as well as a more comprehensive demographic and diagnostic screening, will enable a more valid approach. In addition, like many survey-based studies, the results of this work may be influenced by sample bias. It is possible that individuals who had notably positive or negative experiences with BetterHelp are those who chose to respond to our prompt to participate in research. We are further unable to investigate the effect of the type of psychotherapy provided (ie, cognitive behavioral therapy, acceptance and commitment therapy, etc) on outcomes. However, it is worth noting that the existing literature examining differential effects of different types of psychotherapies for depression does not suggest that psychotherapy type has a significant effect on outcomes in traditional psychotherapeutic settings [8]. In this study, financial and physical heath status were measured by self-report; such subjective indicators may not be fully valid. Future work may seek to utilize additional objective and sensitive measures of financial and physical health status to provide further insight. Finally, in this study, we lacked data on the potentially crucial moderator variables of race, ethnicity, and gender nonconformity.

\section{Conclusions}

Major depressive disorder is a pervasive and debilitating condition from which many individuals are unable to recover due to lack of accessible and appropriate treatment. The existing literature has demonstrated the use of digital technology as a feasible solution to this growing and widespread dilemma. This study examined the effect of a multimodal digital psychotherapy platform for the treatment of depression in adults. We proposed that a multimodal platform through which users can dynamically select from multiple modes of digital communication throughout therapy may be an effective method of delivering psychotherapy to adults with depression. Our study results demonstrate the initial effectiveness of such a model, with users experiencing significant symptom reduction after the intervention. This feasibility study's preliminary demonstration of such a platform's effectiveness provides an important first step in understanding the potential benefits of such a model of mental health care delivery. Given these results, subsequent research will aim to investigate the unique benefits of a preference-based multimodal platform compared with in-person, single- or bimodal psychotherapy dissemination.

\section{Acknowledgments}

ETM is supported by the National Science Foundation Graduate Research Fellowship under grant \#DGE 1752814. The publication is made possible, in part, by support from the Berkeley Research Impact Initiative sponsored by the University of California, Berkeley, Library.

\section{Conflicts of Interest}

ETM is a former consultant for BetterHelp. LN is a community and support manager for BetterHelp.

\section{Multimedia Appendix 1}

BetterHelp video session invitation. 
[PNG File, 157KB-Multimedia Appendix 1]

\section{Multimedia Appendix 2}

BetterHelp live chat invitation.

[PNG File, 53KB-Multimedia Appendix 2]

\section{Multimedia Appendix 3}

BetterHelp live chat interface (user perspective).

[PNG File, 84KB-Multimedia Appendix 3]

\section{Multimedia Appendix 4}

BetterHelp messaging platform.

[PNG File, 156KB-Multimedia Appendix 4]

\section{References}

1. Weissman M, Bland RC, Canino GJ, Faravelli C, Greenwald S, Hwu HG, et al. Cross-national epidemiology of major depression and bipolar disorder. JAMA 1996;276(4):293-299. [Medline: 8656541]

2. Kessler R, Bromet EJ. The epidemiology of depression across cultures. Annu Rev Public Health 2013;34:119-138 [FREE Full text] [doi: 10.1146/annurev-publhealth-031912-114409] [Medline: 23514317]

3. Chaudhury P, Deka K, Chetia D. Disability associated with mental disorders. Indian J Psychiatry 2006 Apr;48(2):95-101 [FREE Full text] [doi: 10.4103/0019-5545.31597] [Medline: 20703393]

4. Druss BG, Hwang I, Petukhova M, Sampson NA, Wang PS, Kessler RC. Impairment in role functioning in mental and chronic medical disorders in the United States: results from the National Comorbidity Survey Replication. Mol Psychiatry 2009 Jul;14(7):728-737 [FREE Full text] [doi: 10.1038/mp.2008.13] [Medline: 18283278]

5. Sayers J. The world health report 2001 - Mental health: new understanding, new hope. Bulletin of the World Health Organisation 2001;79(11) [FREE Full text] [doi: 10.1590/S0042-96862001001100014]

6. Ustün TB, Ayuso-Mateos JL, Chatterji S, Mathers C, Murray CJL. Global burden of depressive disorders in the year 2000. Br J Psychiatry 2004 May;184:386-392. [Medline: 15123501]

7. Dobson KS. A meta-analysis of the efficacy of cognitive therapy for depression. J Consult Clin Psychol 1989 Jun;57(3):414-419. [Medline: 2738214]

8. Cuijpers P, van Straten A, Andersson G, van Oppen P. Psychotherapy for depression in adults: a meta-analysis of comparative outcome studies. J Consult Clin Psychol 2008 Dec;76(6):909-922. [doi: 10.1037/a0013075] [Medline: 19045960]

9. Rupke SJ, Blecke D, Renfrow M. Cognitive therapy for depression. Am Fam Physician 2006 Jan 01;73(1):83-86 [FREE Full text] [Medline: 16417069]

10. Al-Harbi KS. Treatment-resistant depression: therapeutic trends, challenges, and future directions. Patient Prefer Adherence 2012;6:369-388 [FREE Full text] [doi: 10.2147/PPA.S29716] [Medline: 22654508]

11. Rush AJ. STAR*D: what have we learned? Am J Psychiatry 2007 Feb;164(2):201-204. [doi: 10.1176/ajp.2007.164.2.201] [Medline: 17267779 ]

12. Blackburn IM, Bishop S, Glen AI, Whalley LJ, Christie JE. The efficacy of cognitive therapy in depression: a treatment trial using cognitive therapy and pharmacotherapy, each alone and in combination. Br J Psychiatry 1981 Sep;139:181-189. [Medline: 7317698]

13. Rush A, Beck AT, Kovacs M, Hollon S. Comparative efficacy of cognitive therapy and pharmacotherapy in the treatment of depressed outpatients. Cogn Ther Res 1977 Mar;1(1):17-37. [doi: 10.1007/BF01173502]

14. Thomas K, Ellis AR, Konrad TR, Holzer CE, Morrissey JP. County-level estimates of mental health professional shortage in the United States. Psychiatr Serv 2009 Oct;60(10):1323-1328. [doi: 10.1176/ps.2009.60.10.1323] [Medline: 19797371]

15. Aguilera A. Digital Technology and Mental Health Interventions: Opportunities and Challenges. Arbor 2015 May 12;191(771):a210. [doi: 10.3989/arbor.2015.771n1012]

16. Yuen E, Goetter EM, Herbert JD, Forman EM. Challenges and opportunities in internet-mediated telemental health. Professional Psychology: Research and Practice 2012;43(1):1-8. [doi: 10.1037/a0025524]

17. Spek V, Cuijpers P, Nyklícek I, Riper H, Keyzer J, Pop V. Internet-based cognitive behaviour therapy for symptoms of depression and anxiety: a meta-analysis. Psychol Med 2007 Mar;37(3):319-328. [doi: 10.1017/S0033291706008944] [Medline: $\underline{17112400]}$

18. Barak A, Grohol JM. Current and Future Trends in Internet-Supported Mental Health Interventions. Journal of Technology in Human Services 2011 Jul;29(3):155-196. [doi: 10.1080/15228835.2011.616939] 
19. Backhaus A, Agha Z, Maglione ML, Repp A, Ross B, Zuest DD, et al. Videoconferencing psychotherapy: a systematic review. Psychol Serv 2012 May;9(2):111-131. [doi: 10.1037/a0027924] [Medline: 22662727]

20. Kohn R, Saxena S, Levav I, Saraceno B. The treatment gap in mental health care. Bull World Health Organ 2004 Nov;82(11):858-866 [FREE Full text] [Medline: 15640922]

21. Mohr DC, Ho J, Duffecy J, Baron KG, Lehman KA, Jin L, et al. Perceived barriers to psychological treatments and their relationship to depression. J Clin Psychol 2010 Apr;66(4):394-409 [FREE Full text] [doi: 10.1002/jclp.20659] [Medline: 20127795]

22. Berrouiguet S, Baca-García E, Brandt S, Walter M, Courtet P. Fundamentals for Future Mobile-Health (mHealth): A Systematic Review of Mobile Phone and Web-Based Text Messaging in Mental Health. J Med Internet Res 2016 Dec 10;18(6):e135 [FREE Full text] [doi: 10.2196/jmir.5066] [Medline: 27287668]

23. Andersson G, Cuijpers P. Internet-based and other computerized psychological treatments for adult depression: a meta-analysis. Cogn Behav Ther 2009;38(4):196-205. [doi: 10.1080/16506070903318960] [Medline: 20183695]

24. Carlbring P, Andersson G, Cuijpers P, Riper H, Hedman-Lagerlöf E. Internet-based vs. face-to-face cognitive behavior therapy for psychiatric and somatic disorders: an updated systematic review and meta-analysis. Cogn Behav Ther 2018 Jan;47(1):1-18. [doi: 10.1080/16506073.2017.1401115] [Medline: 29215315]

25. Baumeister H, Reichler L, Munzinger M, Lin J. The impact of guidance on Internet-based mental health interventions A systematic review. Internet Interventions 2014 Oct;1(4):205-215. [doi: 10.1016/j.invent.2014.08.003]

26. Lindhiem O, Bennett CB, Rosen D, Silk J. Mobile technology boosts the effectiveness of psychotherapy and behavioral interventions: a meta-analysis. Behav Modif 2015 Nov;39(6):785-804 [FREE Full text] [doi: 10.1177/0145445515595198] [Medline: 26187164]

27. Zickhur K, Smith A. Pew Research Center: Internet \& Technology. 2013 Aug 26. Home Broadband 2013 URL: http://www. pewinternet.org/2013/08/26/home-broadband-2013/ [accessed 2018-11-23] [WebCite Cache ID 748fTFjIV]

28. Watts S, Mackenzie A, Thomas C, Griskaitis A, Mewton L, Williams A, et al. CBT for depression: a pilot RCT comparing mobile phone vs. computer. BMC Psychiatry 2013 Feb 07;13:49 [FREE Full text] [doi: 10.1186/1471-244X-13-49] [Medline: 23391304]

29. Thayer SE, Ray S. Online communication preferences across age, gender, and duration of Internet use. Cyberpsychol Behav 2006 Aug;9(4):432-440. [doi: 10.1089/cpb.2006.9.432] [Medline: 16901247]

30. Mohr D, Tomasino KN, Lattie EG, Palac HL, Kwasny MJ, Weingardt K, et al. IntelliCare: An Eclectic, Skills-Based App Suite for the Treatment of Depression and Anxiety. J Med Internet Res 2017 Dec 05;19(1):e10 [FREE Full text] [doi: 10.2196/jmir.6645] [Medline: 28057609]

31. Aguilera A, Bruehlman-Senecal E, Demasi O, Avila P. Automated Text Messaging as an Adjunct to Cognitive Behavioral Therapy for Depression: A Clinical Trial. J Med Internet Res 2017 Dec 08;19(5):e148 [FREE Full text] [doi: 10.2196/jmir.6914] [Medline: 28483742]

32. Furber G, Jones GM, Healey D, Bidargaddi N. A comparison between phone-based psychotherapy with and without text messaging support in between sessions for crisis patients. J Med Internet Res 2014 Oct 08;16(10):e219 [FREE Full text] [doi: 10.2196/jmir.3096] [Medline: 25295667]

33. Wright J, Wright AS, Albano AM, Basco MR, Goldsmith LJ, Raffield T, et al. Computer-assisted cognitive therapy for depression: maintaining efficacy while reducing therapist time. Am J Psychiatry 2005 Jun;162(6):1158-1164. [doi: 10.1176/appi.ajp.162.6.1158] [Medline: 15930065$]$

34. Hull TD, Mahan K. A Study of Asynchronous Mobile-Enabled SMS Text Psychotherapy. Telemed J E Health 2017 Dec;23(3):240-247. [doi: 10.1089/tmj.2016.0114] [Medline: 27797646]

35. Ogrodniczuk JS, Piper WE, Joyce AS, McCallum M. Effect of patient gender on outcome in two forms of short-term individual psychotherapy. J Psychother Pract Res 2001;10(2):69-78 [FREE Full text] [Medline: 11264331]

36. Ogrodniczuk J, Staats H. [Psychotherapy and gender: do men and women require different treatments? ]. Z Psychosom Med Psychother 2002;48(3):270-285. [Medline: 12136448]

37. Ogrodniczuk J. Differences in Men'S and Women'S Responses to Short-Term Group Psychotherapy. Psychotherapy Research 2004 Jun 01;14(2):231-243. [doi: 10.1093/ptr/kph019]

38. Kirshner L, Genack A, Hauser ST. Effects of gender on short-term psychotherapy. Psychotherapy: Theory, Research \& Practice 1978;15(2):158-167. [doi: 10.1037/h0085856]

39. Frank E, Carpenter LL, Kupfer DJ. Sex differences in recurrent depression: are there any that are significant? Am J Psychiatry 1988 Jan;145(1):41-45. [doi: 10.1176/ajp.145.1.41] [Medline: 3337291]

40. Addis ME, Mahalik JR. Men, masculinity, and the contexts of help seeking. Am Psychol 2003 Jan;58(1):5-14. [Medline: 12674814]

41. Wetherell J, Petkus AJ, Thorp SR, Stein NB, Chavira SA, Campbell-Sills L, et al. Age differences in treatment response to a collaborative care intervention for anxiety disorders. Br J Psychiatry 2013 Jul;203(1):65-72 [FREE Full text] [doi: 10.1192/bjp.bp.112.118547] [Medline: 23580378]

42. Hobbs MJ, Mahoney AEJ, Andrews G. Integrating iCBT for generalized anxiety disorder into routine clinical care: Treatment effects across the adult lifespan. J Anxiety Disord 2017 Oct;51:47-54. [doi: 10.1016/j.janxdis.2017.09.003] [Medline: $\underline{28926805]}$ 
43. Hansen NB, Lambert MJ, Forman EM. The psychotherapy dose-response effect and its implications for treatment delivery services. Clinical Psychology: Science and Practice 2002 Sep;9(3):343. [doi: 10.1093/clipsy.9.3.329]

44. van Drongelen A, Boot CR, Hlobil H, Twisk JW, Smid T, van der Beek AJ. Evaluation of an mHealth intervention aiming to improve health-related behavior and sleep and reduce fatigue among airline pilots. Scand J Work Environ Health 2014 Nov;40(6):557-568 [FREE Full text] [doi: 10.5271/sjweh.3447] [Medline: 25121620]

45. Mattila E, Lappalainen R, Välkkynen P, Sairanen E, Lappalainen P, Karhunen L, et al. Usage and Dose Response of a Mobile Acceptance and Commitment Therapy App: Secondary Analysis of the Intervention Arm of a Randomized Controlled Trial. JMIR Mhealth Uhealth 2016 Jul 28;4(3):e90 [FREE Full text] [doi: 10.2196/mhealth.5241] [Medline: 27468653]

46. Evans W, Nielsen PE, Szekely DR, Bihm JW, Murray EA, Snider J, et al. Dose-response effects of the text4baby mobile health program: randomized controlled trial. JMIR Mhealth Uhealth 2015 Jan 28;3(1):e12 [FREE Full text] [doi: 10.2196/mhealth.3909] [Medline: 25630361]

47. Bowen D, Kreuter M, Spring B, Cofta-Woerpel L, Linnan L, Weiner D, et al. How we design feasibility studies. Am J Prev Med 2009 May;36(5):452-457 [FREE Full text] [doi: 10.1016/j.amepre.2009.02.002] [Medline: 19362699]

48. Singal AG, Higgins PDR, Waljee AK. A primer on effectiveness and efficacy trials. Clin Transl Gastroenterol 2014 Jan 02;5:e45 [FREE Full text] [doi: 10.1038/ctg.2013.13] [Medline: 24384867]

49. Kim SY. Efficacy versus Effectiveness. Korean J Fam Med 2013 Jul;34(4):227 [FREE Full text] [doi: 10.4082/kjfm.2013.34.4.227] [Medline: 23904951]

50. Depression Guideline Team. Michigan Medicine: University of Michigan. 2011 Aug. UMHS Depression Guideline Update URL: http://www.med.umich.edu/1info/FHP/practiceguides/depress/depress.pdf [accessed 2018-11-23] [WebCite Cache ID 748jjuvZE]

51. Nulty D. The adequacy of response rates to online and paper surveys: what can be done? Assessment \& Evaluation in Higher Education 2008 Jun;33(3):301-314. [doi: 10.1080/02602930701293231]

52. Kroenke K, Spitzer RL, Williams JB. The PHQ-9: validity of a brief depression severity measure. J Gen Intern Med 2001 Sep;16(9):606-613 [FREE Full text] [Medline: 11556941]

53. Kroenke K, Spitzer R. The PHQ-9: A New Depression Diagnostic and Severity Measure. Psychiatric Annals 2002 Sep 01;32(9):509-515. [doi: 10.3928/0048-5713-20020901-06]

54. Löwe B, Unützer J, Callahan CM, Perkins AJ, Kroenke K. Monitoring depression treatment outcomes with the patient health questionnaire-9. Med Care 2004 Dec;42(12):1194-1201. [Medline: 15550799]

55. Munder T, Wilmers F, Leonhart R, Linster HW, Barth J. Working Alliance Inventory-Short Revised (WAI-SR): psychometric properties in outpatients and inpatients. Clin Psychol Psychother 2010;17(3):231-239 [FREE Full text] [doi: 10.1002/cpp.658] [Medline: 20013760]

56. BetterHelp. Convenient, affordable, private online counseling, anytime, anywhere URL: https://www.betterhelp.com/ [accessed 2018-05-01] [WebCite Cache ID 6z6Er5RHX]

57. Ross A, Willson V. Paired Samples T-Test. In Basic and Advanced Statistical Tests. Rotterdam: SensePublishers; 2017:17-19.

58. Hedberg EC, Ayers S. The power of a paired t-test with a covariate. Soc Sci Res 2015 Mar;50:277-291 [FREE Full text] [doi: 10.1016/j.ssresearch.2014.12.004] [Medline: 25592936]

59. Howard KI, Kopta SM, Krause MS, Orlinsky DE. The dose-effect relationship in psychotherapy. Am Psychol 1986 Feb;41(2):159-164. [Medline: $\underline{\text { 3516036] }}$

60. Barnett A, van der Pols JC, Dobson AJ. Regression to the mean: what it is and how to deal with it. Int J Epidemiol 2005 Feb;34(1):215-220. [doi: 10.1093/ije/dyh299] [Medline: 15333621]

61. Reid DJ, Reid FJM. Text or talk? Social anxiety, loneliness, and divergent preferences for cell phone use. Cyberpsychol Behav 2007 Jun;10(3):424-435. [doi: 10.1089/cpb.2006.9936] [Medline: 17594267]

62. Forgays D, Hyman I, Schreiber J. Texting everywhere for everything: Gender and age differences in cell phone etiquette and use. Computers in Human Behavior 2014 Feb;31:314-321. [doi: 10.1016/j.chb.2013.10.053]

63. Hamilton KE, Dobson KS. Cognitive therapy of depression: pretreatment patient predictors of outcome. Clin Psychol Rev 2002 Jul;22(6):875-893. [Medline: 12214329]

64. Schlagert HS, Hiller W. The predictive value of early response in patients with depressive disorders. Psychother Res 2017 Dec;27(4):488-500. [doi: 10.1080/10503307.2015.1119329] [Medline: 26776721]

65. Kessing LV, Hansen MG, Andersen PK, Angst J. The predictive effect of episodes on the risk of recurrence in depressive and bipolar disorders - a life-long perspective. Acta Psychiatr Scand 2004 May;109(5):339-344. [doi: 10.1046/j.1600-0447.2003.00266.x] [Medline: 15049770 ]

\section{Abbreviations}

ANCOVA: analysis of covariance

PHQ-9: Patient Health Questionnaire

SMS: short message service

WAI-SR: Working Alliance Inventory-Short Revised 
Edited by G Eysenbach; submitted 02.05.18; peer-reviewed by J Lundgren, W Tumuhimbise; comments to author 07.06.18; revised version received 08.10.18; accepted 26.10.18; published 23.01.19

Please cite as:

Marcelle ET, Nolting L, Hinshaw SP, Aguilera A

Effectiveness of a Multimodal Digital Psychotherapy Platform for Adult Depression: A Naturalistic Feasibility Study

JMIR Mhealth Uhealth 2019;7(1):e10948

URL: http://mhealth.jmir.org/2019/1/e10948/

doi: $10.2196 / 10948$

PMID: $\underline{30674448}$

CEnitan T Marcelle, Laura Nolting, Stephen P Hinshaw, Adrian Aguilera. Originally published in JMIR Mhealth and Uhealth (http://mhealth.jmir.org), 23.01.2019. This is an open-access article distributed under the terms of the Creative Commons Attribution License (https://creativecommons.org/licenses/by/4.0/), which permits unrestricted use, distribution, and reproduction in any medium, provided the original work, first published in JMIR mhealth and uhealth, is properly cited. The complete bibliographic information, a link to the original publication on http://mhealth.jmir.org/, as well as this copyright and license information must be included. 